\title{
Die Rolle der Geographie in der Landschaftsplanung
}

\section{Vorbemerkungen}

Um die Rolle der Geographie in der Landschaftsplanung zu erörtern, müssen wir vorgängig mindestens folgende Fragen beantworten: Was ist Geographie, was Landschaft, was Landschaftsplanung? Auf sie ist nämlich nur mit Alternativen zu reagieren. So wird Geographie wenigstens in $4 \mathrm{Be}-$ deutungen aufgefaßt: als allgemeine Erdwissenschaft, als Erdoberflächen- oder Erdhüllenwissenschaft, als Erdraumwissenschaft oder als Landschaftsforschung, wenn auch die letztgenannte Umschreibung die stichhaltigste ist. ${ }^{1)}$ Ebenso hat der Begriff Landschaft verschiedene Auslegungen. Doch präzisierte er sich im Lauf der Geschichte vom vieldeutigen Ausdruck Region (Gebiet) zum klaren Inhalt «Gruppierung von Litho-, Hydro-, Atmo- und Biosphäre» (inkl. Anthroposphäre), dem freilich zahlreiche Fremdworte (Geosystem, Geochore, Geosynergie u.a.) zugeordnet werden. ${ }^{2)}$ Im Zuge der Entwicklung einer zielbewußten Landschaftspflege wurde allerdings von ihren Betreuern der - anscheinend erfolgreiche - Versuch gemacht, diese klare Begrifflichkeit zugunsten einer engern Auffassung der Landschaft als Natur- oder naturnahes Gebiet einzuschränken. Dieser Versuch kann indes von der Geographie nicht akzeptiert werden, weil triftigste Gründe dagegen sprechen. ${ }^{3)}$ Landschaftsplanung endlich darf als Organisation oder Maßnahme zur Vorbereitung praktischer Gestaltung - und Nutzung - gesunder Landschaften in Form von Plänen und Gesetzen umschrieben werden, wobei sie selbst nur Teil- oder Zwischenglied einer langen Kette von Vorkehren mit dem Ziel objektgemäßer Nutzung ist.

\section{Die Situation der Landschaftsforschung}

Ob nun die Geographie im weiteren Sinn aufgefaßt oder als Landschaftsforschung anerkannt wird, tut der Tatsache keinerlei Abtrag, daß ihr Gegenstand oder Problemkreis Landschaft möglichst umfassend, in seiner vollen Wirklichkeit zu ergründen versucht wird. Dies nötigt, eine etwas einläßlichere Kennzeichnung des Wesens der Landschaft vorauszuschicken. Nach der oben erwähnten Zusammen- setzung aus bestimmten Komponenten handelt es sich bei ihr um ein komplexes Objekt, um ein (offenes) System, mathematisch ausgedrückt, um eine «Menge als Zusammenfassung von besondern wohlunterschiedenen Objekten unserer Anschauung ... zu einem Ganzen» (G.CANTOR). Dieses Ganze ist in bestimmter Weise aufgebaut und unterliegt bestimmten Einflüssen der Zeit und des Raumes. Die Komponenten zeigen eine bestimmte Anordnung: die Bereiche der Lebewesen, der Biosphäre, lagern über den anorganischen Sphären, welche jene aber umgeben. Die Landschaften sind also weder Organismen noch Anorganismen, sondern Kombinationen oder Vergesellschaftungen beider - worin sich übrigens der Unterschied der Geographie von den Natur- und Geisteswissenschaften spiegelt, die erstere gleichsam als Soziologie der Soziologien erscheint (insofern sie die Wechselbeziehungen der verschiedenen Vergesellschaftungen im anorganischen, organischen und anthropischen Bereich zu erfassen trachtet). ${ }^{4)}$

Die Wechselwirkung der Komponenten, aus denen die Landschaften hervorgehen, ist eine räumliche und zeitliche, genauer eine sach-raum-zeitliche. In sachlicher Hinsicht resultieren hieraus ausschließlich aus Naturelementen gebildete Naturlandschaften und Kulturlandschaften, bei welchen der Mensch Mitgestalter, oft Zerstörer ist; beide treten in mannigfachen Varianten auf. In räumlicher Sicht lassen sich Klein- und Großlandschaften, Lokal-, Regional-, Kontinental- und Globallandschaften unterscheiden, wobei allein unter Annahme einer mittleren Größe von $1 \mathrm{~km}^{2}$ Fläche 510 Mio. Einzelgebiete bestehen. Unter dem zeitlichen Gesichtspunkt sondern sich Ur-, Alt- und Junglandschaften, Landschaften des Archaikums, Mesozoikums, Känozoikums wie solche der Geschichte: der Antike, des Mittelalters und der Neuzeit.

Diesem mehr zuständlichen, statischen Gefüge der Landschaft entspricht ein dynamisches, prozessuales. Die Landschaft unterliegt einem ständigen Form-, Stoff- und Energiewechsel, der zu einem quasistationären Gleichgewicht (L.v. BERTALANFFY) tendiert. ${ }^{5)} \mathrm{Zu}$ dieser gewissermaßen inneren Dyna-

Prof. Dr. Ernst Winkler, Mommsenstraße 2, 8044 Zürich. 
mik gesellt sich eine mehr äußere, externe. Die Landschaft bezieht aus der Umwelt (von den andern Landschaften, aus dem Erdinnern und vom Kosmos) Stoffe und Energien und gibt solche dorthin auch ab. Und endlich drückt sich die Dynamik in Landschaftswandlungen, Sukzessionen, Abläufen aus, die progressiv oder regressiv verlaufen. Mit andern Worten die Landschaften entwickeln sich, sie haben eine Geschichte aus der Vergangenheit über die Gegenwart in die Zukunft: Aus Waldlandschaften können Ackerbaugebiete, Steppen, ja Wüsten entstehen und umgekehrt.

Diese nur gedanklich auseinandergehaltenen, konkret meistens kombinierten Aspekte der Landschaft erfordern verschiedene Betrachtungsweisen oder -richtungen. Hier wird von den üblichen (wie der kausal-finalen, analytisch-synthetischen usw.) und von der Untersuchung der Landschaftskomponenten abgesehen. Den strukturellen Aufbau untersucht die Landschaftsmorphologie. Den Vorgängen in der Landschaft widmen sich Landschaftsphysiologie und -chronologie (Landschaftsgeschichte, Genetik); den Beziehungen zur Umwelt geht die Landschaftsökologie nach, die oft auch mit der Landschaftsphysiologie zusammengeworfen wird. In der jüngsten Zeit genießt sie sogar, als Haushaltlehre der Landschaft, eine Vorzugsstellung im Rahmen der Geographie, obwohl sie keineswegs wichtiger ist als die übrigen Disziplinen. Endlich entspringt aus dem Umstand, daß die Landschaften individuelle und typische Eigenschaften aufweisen, die Landschaftstypologie, die klassifikatorische oder systematische Geographie (e. S.). ${ }^{6}$ Sie sucht die Landschaften, analog den systematischen Zweigen der Biologie, Soziologie und anderer Wissenschaften, in eine Ordnung, ein System zu bringen und wird so zur Gesetzeslehre, zur Nomothese der Landschaftsbildung und zur Theorie der Landschaften, wenn ihre Regeln auch immer nur Wahrscheinlichkeits(Probalitäts-)sätze bleiben.

\section{Geographie und Landschaftsplanung}

Diese Hinweise enthalten nun den Schlüssel zur Rolle, Funktion oder Leistung der Geographie in der Landschaftsplanung (und ebenso für Landschaftsgestaltung oder -pflege und -nutzung). Die Geographie als Landschaftsforschung hat diesen praktischen Disziplinen die Erkenntnis- und Verfahrensgrundlagen zu liefern. Allerdings nicht sie allein; das hieße sie überschätzen; denn die Landschaftsforschung selbst ruht zu wesentlichen Teilen auf den Schultern der übrigen Wissenschaften.

Wohl aber hat sie ihnen gegenüber ihre eigene, besondere Aufgabe, aus der Erkenntnis, daß 1. die Landschaften besondere und damit besonders zu erforschende Einheiten, Ausschnitte der Wirklichkeit und deshalb auch eigene Problemkreise sind, und daß sie 2. von keiner anderen Wissenschaft erfaßt werden. Die Geographie hat sich zweifellos dieser Aufgabe nicht immer genügend unterzogen; sie hat vielmehr (und mußte es, genötigt dazu, weil für sie wichtige, aber im Studienbereich anderer Wissenschaften liegende Fragen von diesen unbearbeitet blieben) hie und da auf «fremden Feldern» gepflügt. Das könnte allerdings so gut wie von jeder Wissenschaft gesagt werden und ist überdies nur Ausdruck der Tatsache, daß alle Wissenschaften ein «lebendiges Ganzes» bilden (P.OPPENHEIM)..$^{7}$

Die Geographie war zudem lange eine vornehmlich theoretische Wissenschaft, die außerdem ein wichtiges Hilfsmittel, die Mathematik, vernachlässigte. Sie hat jedoch rechtzeitig erkannt, daß sie weder ausschließliche Theorie noch Selbstzweck sein darf, sondern ihren eigentlichen Sinn im «Dienst am Leben», für die Landschaft und den Menschen sehen muß, der im Beitrag zur Landschaftsplanung, -pflege, und -nutzung wie zur übergeordneten Landesplanung und Raumordnung besteht.

In diesem Beitrag sind eingeschlossen: verstärkte und differenzierte praxisnahe Betrachtungsweisen, z. B. die Berücksichtigung der Nützlichkeit der Landschaft, ihre Eignung (und Nichteignung) für Siedlung, Wirtschaft, Verkehr und Infrastruktur, ihre Belastbarkeit, Empfindlichkeit, Schutzwürdigkeit, die Prüfung der Risiken und Schäden, der Kapazität und der Entwicklungsfähigkeit der Landschaft. Sie beruht auf der intensiven Erforschung der Stoff- und Energieumsätze und der Bilanzen der Landschaftsstrukturen und -funktionen in Vergangenheit, Gegenwart und Zukunft. ${ }^{8)}$

Auf diesen Feststellungen, die im Rahmen der oben beschriebenen Forschungsweisen laufen können, bauen die Arbeiten auf, welche die einzelnen Phasen der Landschaftsplanung markieren: die Landschaftsdiagnose (die Beurteilung des positiven und negativen Verhaltens der Landschaft), die Prognose, die Leitbild- und Modellkonstruktion und die eigentliche Planifikation selbst, die in den Plänen und den dazugehörigen Gesetzen gipfelt.

Innerhalb dieser Phasen spielt die Ausarbeitung der Pläne die Hauptrolle. Sie kommt in Teil- und Gesamtplänen, Sach-, Raum- und Zeitplänen zum Ausdruck. Inbezug auf die Sachgruppierung unterscheidet man Bau- und Freiflächenpläne, Landwirtschaftszonen-, Wald-, Erholungs-, Abbau- und Landschaftsschutzpläne, Pläne des Unproduktivoder Ödlandes (Fels- und Schuttgebiete, Gletscher usw.) und der Gewässer sowie Gefahrenpläne (Lawinen, Rutsche usw.). Hinsichtlich der Raumdimension sind Orts-, Gemeinde-, Regional-, Kantonal- und Landes- oder Bundespläne üblich. Bezüglich der Zeit gelten Kurz-, Mittel- und Langfrist- 
pläne. Zusätzlich werden Richt-, Gestaltungs- und Nutzungspläne unterschieden. Die Richtpläne sind bekanntlich beschränkt verbindlich, während Nutzungspläne generelle Gültigkeit haben.

Für sie alle kann die Landschaftsforschung wesentliche Unterlagen bereitstellen und sachliche Impulse liefern, seien es auf die bestehende und künftige Bevölkerung bezogene Bedarfszahlen des Bauens, des Verkehrs und der Wirtschaft, sowie Richtwerte oder methodische Richtlinien. Dabei erweist sich für die Pläne die Koordination bzw. Aufeinanderabstimmung (beispielsweise mittels Vorrangermittlungen) als wichtigstes Entscheidungskriterium. Sie bedarf besonders subtiler mathematischer wie intuitiver Fundierung.

Da dem Fernziel der Landschafts- und Landesplanung wie der Raumordnung in der Regel mannigfache Hindernisse, wirtschaftliche, (besonders eigentums-) rechtliche und politische Widerstände entgegentreten, ergeben sich weitere Probleme, die von der Landschaftsforschung angegangen werden müssen und können. Mit der Aufstellung der Pläne ist es übrigens nicht getan; sie sind durch Planungskommentare, Reglemente und Gesetze zu ergänzen, welche für die Realisierung sogar die entscheidenden Phasen darstellen. Auch für sie kann die Landschaftsforschung maßgebliche Ratschläge suchen, wiewohl diese Arbeit bereits in die Nähe juristischer Überlegungen führt.

Und da die Planung eine unaufhörliche Aufgabe ist, weil sich sowohl die Landschaftsobjekte wie die Ansprüche an sie und damit auch die gestalterischen wie die nutzenden Maßnahmen ständig wandeln, bildet die stetige Revision aller planerischen Handlungen ein grundlegendes Glied im Gesche- hen. Damit wird auch die Landschaftsforschung zur Daueraufgabe im Rahmen der Planung, so wie diese an sich innerhalb der gesamten menschlichen Tätigkeit bereits eine solche ist.

\section{Ausblick}

Damit ist die Rolle der Geographie in der Landschaftsplanung und - das sei wiederholt - der Landschaftspflege und -nutzung in den gröbsten Zügen umrissen. Auf eine Differenzierung und Systematisierung wurde mit Absicht verzichtet, da sowohl Landschaftsplanung als auch Landschaftsforschung trotz bereits jahrzehntelangen Bemühens sich noch weitgehend im Anfangsstadium bewegen und darum raschen Änderungen unterworfen sind. Wohl aber darf abschließend der Wunsch geäußert werden, daß sie sich zunehmend enger verbinden, damit ihre gemeinsamen Objekte, die Landschaften, einer immer positiveren Gestaltung und Nutzung entgegengeführt werden können.

\section{Résumé}

La géographie, prise au sens large ou étroit, est toujours une importante science fondamentale pour l'aménagement du paysage ainsi que pour l'aménagement du territoire. Elle doit mettre à disposition le matériel de base lors du relevé du paysage et aider à maîtriser les principes et les données (Indices) lors de différentes phases de travail. Par là elle devient une discipline égale en droits, et elle a encore beaucoup de possibilités de se développer.
1) Diese Ansicht darf wohl trotz vielfacher, «moderner» anderer Meinungen nach wie vor volle Geltung beanspruchen. Sie kommt u.a. in der auf H. HASSINGER, im Grunde auf A.v. HUMBOLDT und C.RITTER zurückgeführten Ansicht C. TROLLs zum Ausdruck, der im Aufsatz «Die Landschaft und ihre Erforschung» sagt: «Mit der Landschaft hat die Geographie ... endlich ... ihr eigenes Objekt gefunden, das ihr keine andere Wissenschaft streitig machen kann, während die Beschäftigung mit einzelnen Faktoren sie mit vielen Nachbarfächern . . . in lebendiger Berührung halten kann» (Studium Generale 3, 1950, S. 163).

2) Mit dieser Begriffsbestimmung dürfte das Wesen der Landschaft eindeutiger und objektgemäßer umschrieben sein als mit den üblichen Raum- oder Regionaldefinitionen (Landschaft als Erdraum, Gebiet usw.).

3) Vor allem, weil die sogenannten «naturnahen» Landschaften von so zahlreichen anthropogenen Elementen: Siedlungen, Verkehrswegen, landwirtschaftlichen Nutzungen durchsetzt sind, daß ihre eindeutige Unterscheidung von den Kulturlandschaften unmöglich erscheint.

4) W.TOMASEK: Landschaft als System. Hannover 1968 G.CANTOR: Gesammelte Abhandlungen mathematischen und philosophischen Inhalts. Hildesheim 1895, S. 282; E. NEEF Die theoretischen Grundlagen der Landschaftslehre. Gotha 1967; D. HARVEY: Explanation in Geography. London 1969; J.G.SAUSCHKIN: Studien zu Geschichte und Methodologie der geographischen Wissenschaft. Gotha 1978; E.WIRTH: Theoretische Geographie. Stuttgart 1979.

5) L.v. BERTALANFFY: Vorläufer und Begründer der Systemtheorie. Berlin 1972, S. 17 ff. und General System Theory. London 1971.

6) Dieses Betrachtungssystem wurde erstmals entwickelt in «Was ist Geographie». Bildung 1, 1934, S. 13 f. Es fand Eingang bei C.TROLL a.a.O. S. 166 ff., bei G. FOCHLER-HAUKE: Geographie, Frankfurt a.M. 1968, S. 234 ff., bei E. NEEF: Das Gesicht der Erde, Leipzig 1975, S. 701 u. a.

7) P.OPPENHEIM: Die natürliche Ordnung der Wissenschaften. Jena 1926, S. 1 .

8) Vgl. H.GUTERSOHN: Die Geographie als Grundlage der Orts-, Regional- und Landesplanung. Basler Beiträge zur Geographie und Ethnologie H. 5, 1962; J. JACSMAN: Einführung in die Landschaftsplanung. Zürich 1967; J. JACSMAN und E. WINKLER: Zur Methodik der Landschaftsplanung. Plan 25, 1968, S.134-138; F.MULLER: Physische Geographie und Raumplanung und W. A. GALLUSSER: Geographie und Landschaftsplanung. Beide in: Der ländliche Raum. Eine Aufgabe der Raumplanung. Zürich 1977, S. 25 ff.; C.u. S. JELLICOE: The Landscape of Man. London 1975; K. BUCHWALD und W. ENGELHARDT: Handbuch für Landschaftspflege und Naturschutz. München 1968/69; B. HACKETT: Landscape Planning. Newcastle upon Tyne 1971 